This is the accepted version of the article:

Santidrián A., Kierkowicz M., Pach E., Darvasiová D., Ballesteros B., Tobias G., Kalbác M.. Charge transfer in steam purified arc discharge single walled carbon nanotubes filled with lutetium halides. Physical Chemistry Chemical Physics, (2020). 22. : 10063 - . 10.1039/d0cp01408g.

Available at: https://dx.doi.org/10.1039/d0cp01408g 


\title{
CHARGE TRANSFER IN STEAM PURIFIED ARC DISCHARGE SINGLE WALLED CARBON NANOTUBES FILLED WITH LUTETIUM HALIDES.
}

\author{
Ana Santidrián ${ }^{1}$, Magdalena Kierkowicz ${ }^{2}$, Elzbieta Pach ${ }^{3}$, Denisa Darvasiová \\ 1,a, Belén Ballesteros ${ }^{3}$, Gerard Tobias ${ }^{2}$, Martin Kalbáč ${ }^{1}$. \\ ${ }^{1}$ J. Heyrovsky Institute of the Physical Chemistry, Dolejskova 3, 18223 Prague 8, Czech Republic \\ ${ }^{2}$ Institut de Ciencia de Materials de Barcelona (ICMAB-CSIC), Campus UAB, Bellaterra 08193, Barcelona, \\ Spain.
}

${ }^{3}$ Catalan Institute of Nanoscience and Nanotechnology (ICN2), CSIC and The Barcelona Institute of Science and Technology, Campus UAB, Bellaterra, 08193 Barcelona, Spain.

a Current address: Institute of Physical Chemistry and Chemical Physics, Slovak University of Technology, Radlinskeho 9, 81237 Bratislava, Slovakia

\section{ABSTRACT}

In the present work, the effect of doping on electronic properties in bulk purified and filled arc-discharge single-walled carbon nanotubes samples is studied for the first time by in situ Raman spectroelectrochemical method. A major challenge to turn the potential of SWCNTs into customer applications is to reduce or eliminate their contaminants by means of purification techniques. Besides, the endohedral functionalization of SWCNTs with organic and inorganic materials (i.e. metal halides) allows the development of tailored functional hybrids. Here, we report the purification and endohedral functionalization of SWCNTs with doping affecting the SWCNTs. Steam-purified SWCNTs have been filled with selected lutetium(III) halides, $\mathrm{LuCl}_{3}, \mathrm{LuBr}_{3}, \mathrm{LuI}_{3}$, and sealed using high-temperature treatment, yielding closed-ended SWCNTs with the filling material confined in the inner cavity. The purified SWCNTs were studied using TGA, EDX, STEM and Raman spectroscopy. The lutetium(III) halide-filled SWCNTs (LuX $@$ @SWCNTs) were characterized using STEM, EDX, Raman spectroscopy and in situ Raman spectroelectrochemistry. It was found that there is a charge transfer between the SWCNTs and the encapsulated $\mathrm{LuX}_{3}(\mathrm{X}=\mathrm{Cl}, \mathrm{Br}, \mathrm{I})$. The obtained data testify to the acceptor doping effect of lutetium(III) halides incorporated into the SWCNT channels, which is accompanied by the charge transfer from nanotube walls to the introduced substances. 


\section{INTRODUCTION}

Single-walled carbon nanotubes (SWCNTs) have many potential applications that require processing techniques such as purification of amorphous carbon, graphitic particles and metal catalyst particles. ${ }^{1}$ Purification is also essential for filling SWCNTs with a chosen payload (endohedral functionalization) and/or external functionalization (exohedral functionalization). ${ }^{2-5}$

Functionalization of SWCNTs, endohedral or exohedral, with compounds in which electrons or holes are introduced into their electronic structure, causes an increase or decrease of the electron density on the nanotube walls, respectively, and, therefore, allows us to control the electronic properties of the system. ${ }^{5}$ Filling of SWCNTs is used to produce novel electronic devices, nanosensors and inner cavities for endohedral catalysis. ${ }^{6-9}$ The electronic structure can be modified via $n$ - or $p$-type doping with alkali metals, chalcogenides, organic and organometallic compounds, and other materials. ${ }^{10-14}$ In this way, doping experiments are suitable for changing, and also tailoring, the electronic structure of the SWCNTs. Despite this, a method to study the effect of the endohedral functionalization on the electronic structure of SWCNTs is needed.

In this work, we use Raman spectroscopy as it is one of the most powerful characterization methods because it is a non-destructive, contactless, quick technique with relatively simple or no preparation required, and it is sensitive to changes in physical and chemical properties of SWCNTs. ${ }^{15,16}$ The Raman spectra of SWCNTs present several features. Beginning from small Raman shifts, the main features of the Raman spectrum of SWCNTs are the low-energy modes observed between 100 and 400 $\mathrm{cm}^{-1}$, the so-called radial breathing modes (RBM) where all atoms of the tube vibrate radially in phase. Noteworthy, the energy of these vibrational modes depends on the diameter of the SWCNTs. Following the Raman shifts, the tangential modes are the most intense high-energy modes of SWCNTs and form the so-called G-band which is observed at around $1600 \mathrm{~cm}^{-1}$. The line shape of the G-band can be used to distinguish between metallic and semiconducting SWCNTs. ${ }^{17,18}$ In addition, for samples containing structural defects, the disorder-induced mode (D-band) is observed at around $1350 \mathrm{~cm}^{-1} .{ }^{19}$ The level of doping of the SWCNTs can easily be adjusted by applying a potential. Electrochemical charging can be understood using a double-layer capacitor model where the SWCNTs act as the working electrode and the charge carriers are injected into the SWCNTs, while the electrolyte ions compensate the charge. In that way, extra electrons or holes are inserted in the SWCNTs causing changes in the electronic structure and these changes can be followed using Raman spectroscopy (in situ Raman spectroelectrochemistry). ${ }^{20,21}$ In light of the above, in situ Raman spectroelectrochemistry, is an important tool for the characterization of SWCNT samples. In the present report, we show a comprehensive picture of the influence of the endohedral functionalization of SWCNTs with lutetium(III) halides (hereinafter called LuX 3 @SWCNT, X $=\mathrm{Cl}$, Br, or I) on the electronic structure of 
the nanotubes. Raman spectroscopy, in particular the G-band, is usually used to characterize the electronic structure of nanocarbon samples. ${ }^{22}$ However, the changes in the G-band are sometimes masked by other effects, such as strain. Consequently, we used here a novel approach employing in situ Raman spectroelectrochemical method to determine the type of doping in the purified and filled SWCNT samples.

Metal halides were employed as filling in the present study because their encapsulation into nanotubes also finds application, for instance, as radiotracers in the biomedical field. ${ }^{3,23} \mathrm{LuX}_{3}$ are nonradioactive analogues of radioactive ${ }^{177} \mathrm{LuX}_{3}$. Scanning transmission electron microscopy (STEM), energy-dispersive X-ray spectroscopy (EDX) and Raman spectroscopy were employed to elucidate different aspects of the interaction between SWCNTs and lutetium(III) halides. The charging behaviour of the Raman shift in LuX $@$ SWCNT was studied using in situ Raman spectroelectrochemistry.

\section{EXPERIMENTAL}

Arc-discharge SWCNTs were purchased from Carbon Nanotechnologies, Inc. Lutetium(III) chloride, lutetium(III) bromide and lutetium(III) iodide were purchased from Sigma-Aldrich (anhydrous, $\leq 99.9 \%$ trace metals basis).

SWCNTs were initially steam and $\mathrm{HCl}$ treated, before being filled with the chosen lutetium(III) halide. The steam treatment was performed following the protocol that has been previously reported for the purification and shortening of chemical vapour deposition (CVD)-grown SWCNTs. ${ }^{1,24}$ Raw arcdischarge SWCNTs were ground in an agate mortar and pestle and spread inside a silica tube $(4 \mathrm{~cm}$ in diameter). The silica tube was placed inside an alumina tube $(5 \mathrm{~cm}$ in diameter $)$ in the centre of a tubular furnace. Steam was introduced at a rate of $0.58 \mathrm{~mL} / \mathrm{min}$ by purging a continuous flow of argon (Carburos Metálicos, 99.999\%) through hot water $\left(98^{\circ} \mathrm{C}\right)$. The system was initially purged with Ar for $2 \mathrm{~h}$ to ensure the complete removal of air. Then, the furnace was annealed at a rate of $30^{\circ} \mathrm{C} / \mathrm{min}$ and held at $900{ }^{\circ} \mathrm{C}$ for selected periods of time: $0.5 \mathrm{~h}, 1 \mathrm{~h}, 2 \mathrm{~h}, 3 \mathrm{~h}, 4 \mathrm{~h}$ and $5 \mathrm{~h}$. It has been observed in samples of CVD-grown SWCNTs that steam removes the amorphous carbon and graphitic shells that sheathe catalytic metal particles employed for the growth of the CNTs. ${ }^{1,24}$ Therefore, each of the steamtreated samples was independently refluxed with $6 \mathrm{M} \mathrm{HCl}$ to dissolve the exposed metal particles. The sample was collected by filtration onto polycarbonate membrane $\left(0.2 \mu \mathrm{m}\right.$ pore size, Whatman ${ }^{\circledR}$ Nucleopore) and rinsed with distilled water until the $\mathrm{pH}$ of the filtrate was neutral. The collected solid powder was dried overnight at $100^{\circ} \mathrm{C}$.

Thermogravimetric analysis (TGA) of the raw and purified samples was performed in a NETZSCH-STA 449 F1 Jupiter instrument. Samples were annealed under flowing air at a heating rate of $10{ }^{\circ} \mathrm{C} / \mathrm{min}$ until $900^{\circ} \mathrm{C}$. 
pSWCNTs (4h steam $+\mathrm{HCl}$ purification) were filled with lutetium(III) chloride $\left(\mathrm{LuCl}_{3}\right)$, lutetium(III) bromide ( $\left.\mathrm{LuBr}_{3}\right)$ and lutetium(III) iodide $\left(\mathrm{LuI}_{3}\right)$ by molten-phase filling. pSWCNTs (400 $\mathrm{mg}$ ) and a given metal halide $\left(\mathrm{LuX}_{3}\right)$ were ground together in a weight ratio of 1:10 (pSWCNTs:LuX $)_{3}$ inside an argon-filled glove box until the mixture presented a uniform colour. The sample was placed inside a silica tube and sealed under vacuum before annealing at $940{ }^{\circ} \mathrm{C}$ for $\mathrm{LuCl}_{3}, 1050{ }^{\circ} \mathrm{C}$ for $\mathrm{LuBr}_{3}$ and $1100{ }^{\circ} \mathrm{C}$ for $\mathrm{LuCl}_{3}$ for $12 \mathrm{~h}$. Once the process was finished, to remove the excess of $\mathrm{LuX}_{3}$, external to the nanotubes, the collected sample was dispersed in distilled water, placed inside a dialysis sack and washed with water in combination with a Soxhlet system. ${ }^{25}$ The resulting samples of filled tubes, named as LuX $3 @$ pSWCNTs $(\mathrm{X}=\mathrm{Cl}, \mathrm{Br}, \mathrm{I})$, were recovered by filtration onto a polycarbonate membrane. The samples were dried overnight at $100{ }^{\circ} \mathrm{C}$.

Raman spectra were acquired using a LabRAM HR Raman spectrometer (Horiba Jobin-Yvon), and laser excitation energies of 2.54 and $2.33 \mathrm{eV}$ (488 and $532 \mathrm{~nm}$, respectively, $\mathrm{Ar} / \mathrm{Kr}$ laser, Coherent) and of $1.96 \mathrm{eV}(633 \mathrm{~nm}, \mathrm{He}-\mathrm{Ne})$. A $50 \times$ objective was used with a laser spot of about $1 \mu \mathrm{m}$. The laser power was $1 \mathrm{~mW}$ and the spectral resolution was $1 \mathrm{~cm}^{-1}$. Each sample was measured in multiple regions. Raman mapping was conducted with lateral steps of $1 \mu \mathrm{m}$ (both in the $X$ and $Y$ directions) on rectangular areas with varying sizes (49 data points or 900 data points for each map). The samples were measured under ambient conditions.

The Raman spectroelectrochemistry was performed in a three-electrode cell assembled in a glove box. The measured nanotubes were cast on a Pt wire from their dispersion in methanol, which was employed as the working electrode. Another Pt wire was employed as a counter electrode and a Ag wire as a reference electrode. An Autolab PGSTAT (Ecochemie) potentiostat was used to apply potential. The electrolyte solution was $\mathrm{LiClO}_{4}(0.2 \mathrm{M})$ dissolved in dry acetonitrile. The potential was ramped to $\pm 1.5 \mathrm{~V}$ in steps of $0.3 \mathrm{~V}$, and Raman spectra were acquired at a constant potential at every step. The high-frequency component of the $\mathrm{G}^{-}$band, the $\mathrm{G}^{+}$, was fitted by a Lorentzian line shape, whereas the low-frequency component, the $\mathrm{G}^{-}$, was fitted using a Breit-Wigner-Fano (BWF) line shape. The D-band was fitted using a Lorentzian line shape.

\section{RESULTS AND DISCUSSION}

\section{Purification of arc-discharge SWCNTs}

For the first time, steam purification process was performed on raw arc-discharge SWCNTs. The raw SWCNTs were treated with steam at $900{ }^{\circ} \mathrm{C}$ for periods of $0.5,1,2,3,4$ and $5 \mathrm{~h}$. The steamtreated samples were then independently treated with $6 \mathrm{M} \mathrm{HCl}$. TGA was carried out under flowing air to investigate the amount of metal catalyst present in the samples before and after the purification treatment (Electronic Supporting Information Figure ESI1). EDX analysis of the inorganic solid residue 
collected after TGA of raw SWCNTs under oxidizing conditions reveals the presence of iron and oxygen (Electronic Supporting Information Figure ESI2). This indicates that iron acted as a catalyst for the growth of the SWCNTs. Assuming that iron is the only inorganic impurity present in raw SWCNTs, it is possible to determine its content in each of the samples from the TGA residue, which corresponds to $\mathrm{Fe}_{2} \mathrm{O}_{3}$. As can be seen in Figure 1a, a dramatic decrease of the iron content is observed by treating the raw material ( $30.1 \mathrm{wt} \%$ solid residue; $21.1 \mathrm{wt} \% \mathrm{Fe})$ with $0.5 \mathrm{~h}$ steam and $\mathrm{HCl}(12.4 \mathrm{wt} \%$ solid residue; $8.7 \mathrm{wt} \% \mathrm{Fe}$ ). Longer steam treatments resulted in a further decrease in the metal content to 2.9 $\mathrm{wt} \% \mathrm{Fe}(4.2 \mathrm{wt} \%$ solid residue) after $4 \mathrm{~h}$ steam and $\mathrm{HCl}$. It is worth noticing that increasing the steam treatment to $5 \mathrm{~h}$ does not significantly alter the total amount of inorganic impurities. Steam purification is an extremely simple and successful strategy. However, the production yield is low (around $10 \%$ ). The reason is because steam reacts through the ends of SWCNTs and each long SWCNT results in a corresponding short SWCNT. The steam treatment goes through the following reactions: ${ }^{27}$

$\mathrm{C}_{(\mathrm{s})}+\mathrm{H}_{2} \mathrm{O}(\mathrm{g}) \rightarrow \mathrm{CO}_{(\mathrm{g})}+\mathrm{H}_{2}(\mathrm{~g})$

$\mathrm{CO}_{(g)}+\mathrm{H}_{2} \mathrm{O}_{(g)} \rightarrow \mathrm{CO}_{2}(g)+\mathrm{H}_{2}(g)$

Therefore, from TGA analysis, $4 \mathrm{~h}$ steam (and $\mathrm{HCl}$ ) is the optimal purification protocol for the subsequent filling experiments (sample named pSWCNTs).

Next, Raman spectroscopy with three different excitation energies, $1.96 \mathrm{eV}(633 \mathrm{~nm}), 2.33 \mathrm{eV}$ $(532 \mathrm{~nm})$ and $2.54 \mathrm{eV}(488 \mathrm{~nm})$, was performed to understand the influence of steam on the physical and structural properties of the arc-discharge SWCNTs (Electronic Supporting Information Figure ESI3). To evaluate the number of defects in the samples, in Figure $1 \mathrm{~b}$ the intensity ratio between $\mathrm{D}$ and G-bands $\left(I_{\mathrm{D}} / I_{\mathrm{G}}\right.$ ratio $)$ is represented. As observed in Figure $1 \mathrm{~b}$, the $I_{\mathrm{D}} / I_{\mathrm{G}}$ ratio decreases as the time of treatment increases. This correlation is consistent with the fact that amorphous carbon present in raw SWCNTs is removed during the steam treatment along with the most defective SWCNTs. Besides, studying the decreasing tendency of the $I_{\mathrm{D}} / I_{\mathrm{G}}$ ratio with the time of treatment, we can observe that no significant number of new defects was created. In agreement with the Raman analysis, STEM inspection of the steam-treated SWCNTs also reveals that the morphology of the SWCNTs has been well preserved after the steam treatment (Electronic Supporting Information Figure ESI4) and the number of catalytic particles, visible as bright dots, has been substantially reduced. This confirms that steam is highly efficient in removing carbonaceous impurities and catalytic particles from raw arc discharge SWCNT samples, and thus the steam purification was successful. Length characterization of both raw and purified arc-discharge SWCNTs was also determine (Electronic Supporting Information ESI5). ${ }^{26}$ 
a.

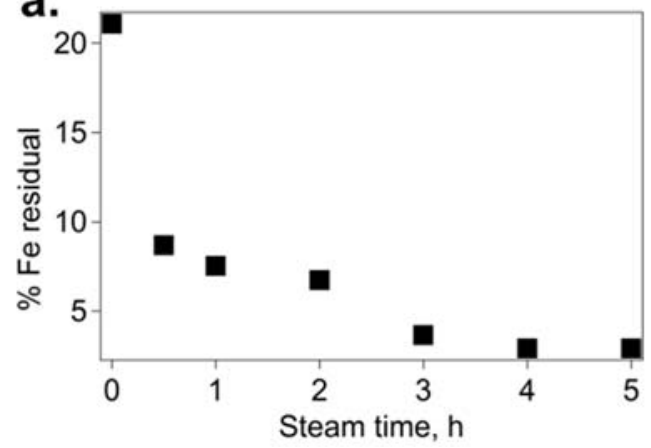

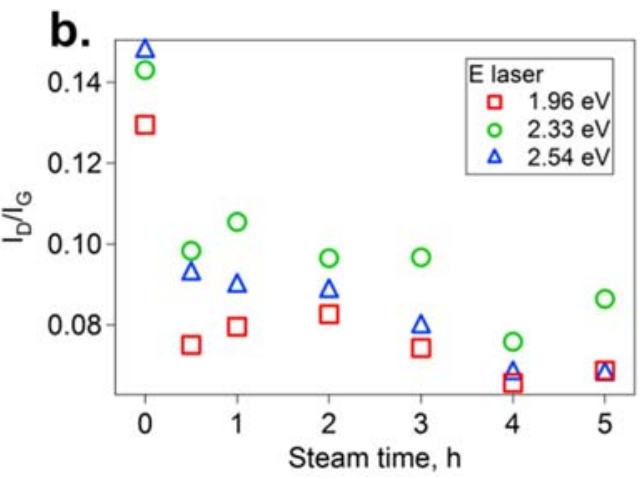

Figure 1: a. Amount of iron calculated from the inorganic solid residue (determined by TGA) in the raw SWCNTs $(0 \mathrm{~h})$ and steam-treated samples during $0.5,1,2,3,4$ and $5 \mathrm{~h}$ (after an $\mathrm{HCl}$ wash). b. $I_{\mathrm{D}} / I_{\mathrm{G}}$ ratio of the raw SWCNTs $(0 \mathrm{~h}$ treatment) and steam-treated SWCNTs for different periods of time (after an $\mathrm{HCl}$ wash). The samples were excited using $1.96 \mathrm{eV}$ (red squares), $2.33 \mathrm{eV}$ (green circles) and 2.54 $\mathrm{eV}$ (blue triangles) laser energies.

\section{Filling and washing of arc-discharge SWCNTs}

Because SWCNTs with a low degree of structural defects are of interest for the growth and shielding of one-dimensional compounds, we filled the sample of pSWCNTs (4h steam $+\mathrm{HCl}$ ) with lutetium(III) halides: lutetium(III) chloride $\left(\mathrm{LuCl}_{3}\right)$, lutetium(III) bromide $\left(\mathrm{LuBr}_{3}\right)$ and lutetium(III) iodide $\left(\mathrm{LuI}_{3}\right)$. The metal halide was encapsulated by molten-phase high-temperature filling, which results in the formation of filled closed-ended CNTs. ${ }^{27}$ Having closed ends allows the removal of the external material while preserving the encapsulated compounds. ${ }^{25,27}$ The encapsulation of the metal halides into SWCNTs was confirmed by electron microscopy imaging and spectroscopy.

HAADF-STEM were acquired on a SEM operated at $20 \mathrm{kV}$ (Figure 2). Imaging at low operating voltages prevents damage to the carbon nanotubes by electron beam irradiation. HAADFSTEM imaging is a powerful tool that allows the direct visualization of the encapsulated material. Because the contrast formation of the image is approximately proportional to the square of the atomic number of the element, heavy elements such as lutetium appear much brighter than carbon from the SWCNTs. Figure 2 shows STEM images of the resulting samples where filled carbon nanotubes can be clearly seen. The bright lines observed on the images correspond to $\mathrm{LuCl} 3, \mathrm{LuBr} 3$ and LuI3. These bright lines follow the shape of the SWCNTs (appearing as pale grey), an indication of the successful encapsulation of the payloads. To confirm further the presence of the metal halides $\left(\mathrm{LuCl}_{3}, \mathrm{LuBr}_{3}\right.$ and $\mathrm{LuI}_{3}$ ) and assess the purity of the prepared samples, EDX spectra were acquired using a transmission electron microscope (Electronic Supporting Information ESI7). 


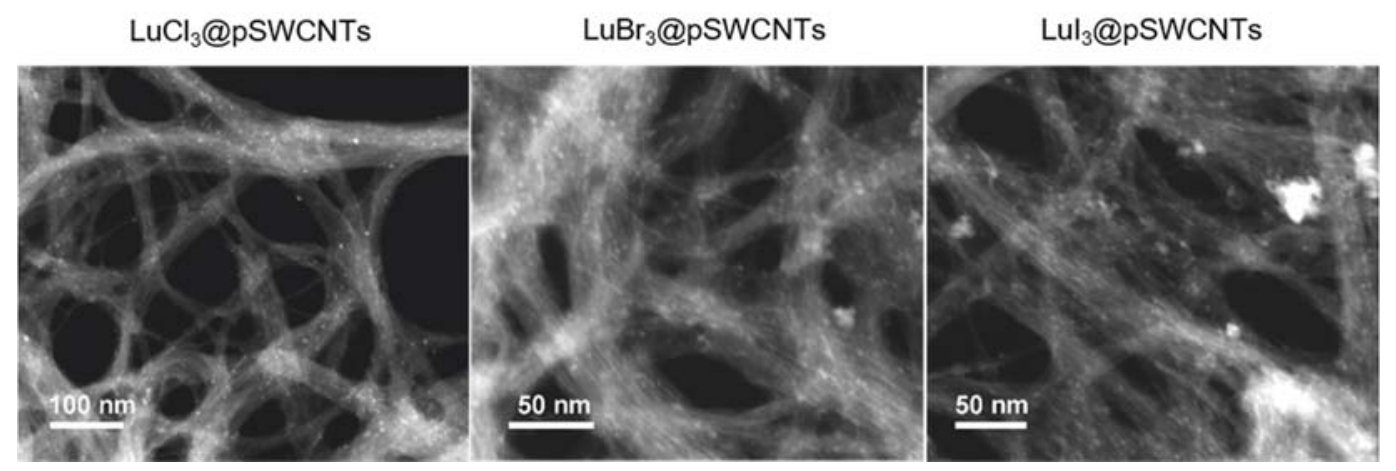

Figure 2: HAADF-STEM analysis (SEM) of pSWCNTs (4 h steam $+\mathrm{HCl}$ treatment) filled with $\mathrm{LuCl}_{3}$, $\mathrm{LuBr}_{3}$ and $\mathrm{LuI}_{3}$.

Using Raman spectroscopy, we studied the filling of SWCNTs $\left(\mathrm{LuCl}_{3} @ \mathrm{pSWCNTs}\right.$, $\mathrm{LuBr}_{3} @$ pSWCNTs and LuI $@$ @pSWCNTs). The pSWCNTs were used as a control sample. SWCNTs with different diameters are in resonance at different laser energies. Therefore, pSWCNTs, LuCl ${ }_{3} @$ pSWCNTs, LuBr $3 @$ pSWCNTs and LuI $@$ pSWCNTs were measured using three different excitation energies: $2.54 \mathrm{eV}(488 \mathrm{~nm}), 2.33 \mathrm{eV}(532 \mathrm{~nm})$ and $1.96 \mathrm{eV}(633 \mathrm{~nm})$ to examine how the different fillings affect tubes with different diameters $\left(\mathrm{d}_{\mathrm{t}}\right)$. Figure $3 \mathrm{a}-\mathrm{c}$ shows the RBM regions and Figure $3 \mathrm{~d}-\mathrm{f}$ shows the $\mathrm{G}$ regions of the Raman spectra of pSWCNTs, LuCl $@$ pSWCNTs, LuBr $3 @$ pSWCNTs and LuI $@$ pSWCNTs excited by the following laser lines: 2.54 (a and d), 2.33 (b and e) and $1.96 \mathrm{eV}$ (c and f), respectively.
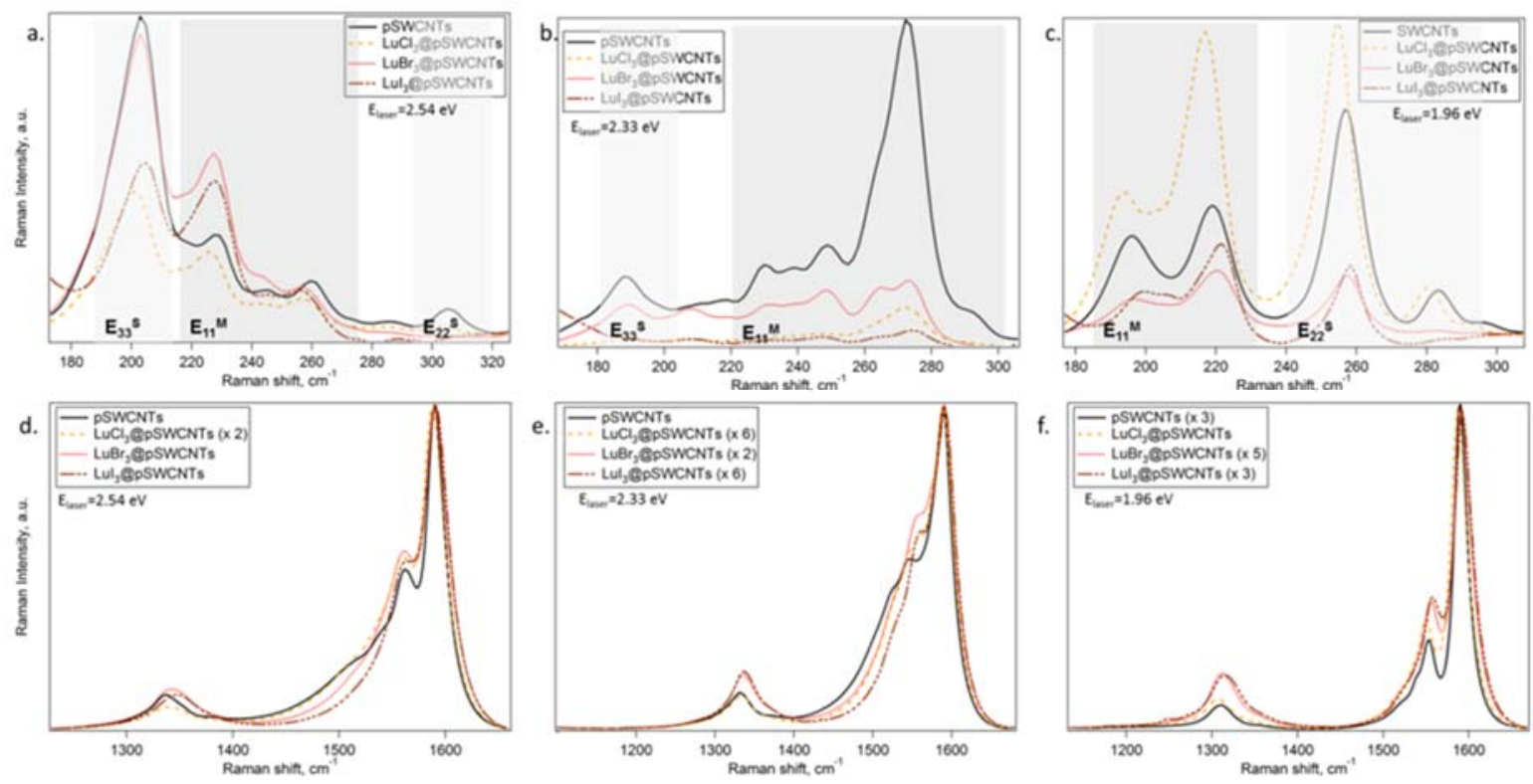

Figure 3: Resonance Raman spectra of the RBM region (top) and the D-band and G-band region (bottom) of pSWCNTs (black line), LuCl $@$ @pSWCNTs (yellow dotted line), LuBr 3 @pSWCNTs (red line) and LuI ${ }_{3} @$ pSWCNTs (brown dashed line) excited by different laser lines (a, d) 2.54 eV, (b, e) $2.33 \mathrm{eV}$ and $(\mathrm{c}, \mathrm{f}) 1.96 \mathrm{eV}$. In Figure $3(\mathrm{a}, \mathrm{b}, \mathrm{c})$ the grey and black regions correspond to the $E_{33} \mathrm{~s}, E_{22} \mathrm{~s}$ and $E_{11}{ }^{\mathrm{M}}$ electronic transitions, respectively. Each spectrum is an average based on $900(1.96 \mathrm{eV})$ or 49 
spectra $(2.33$ and $2.54 \mathrm{eV})$ measured in different points, and are multiplied by the factor as specified in the legend.

Figure $3 \mathrm{a}-\mathrm{c}$ shows the RBM region of the Raman spectra of SWCNTs excited by 2.54, 2.33 and $1.96 \mathrm{eV}$, respectively. The positions of the most intense RBM bands in each region are recorded in Table 1. Only the SWCNTs whose transition energies (denoted as $E_{\mathrm{ii}}$ ) are in resonance with (or close to) the $E_{\text {laser }}$ show RBM bands in the Raman spectrum. The electronic transitions were assigned using the Kataura plot. ${ }^{28}$ Besides, using the RBM frequency ( $\omega_{\text {RBM }}$ ), the diameter of SWCNTs $\left(\mathrm{d}_{\mathrm{t}}\right)$ was estimated. ${ }^{29}$ Figure $3 \mathrm{a}, \mathrm{b}$ and $\mathrm{c}$ is divided into three regions where each range of the RBM modes corresponds to tubes excited via the $E_{33} \mathrm{~s}, E_{11}{ }^{\mathrm{M}}$ and $E_{22} \mathrm{~s}$ electronic transitions. Hence, both semiconducting (S) and metallic (M) SWCNTs are in resonance with the 2.54, 2.33 and $1.96 \mathrm{eV}$ excitation energies used.

Table 1: Diameter distribution calculated from the RBM Raman shift in the Raman spectra of pSWCNTs acquired at different laser excitation wavelengths of the most intense RBM bands using $\omega_{\text {RBM }}$ and assignment of the electronic transitions using the Kataura plot. ${ }^{28}$

\begin{tabular}{|c|c|c|c|c|c|c|c|c|}
\hline \multicolumn{3}{|c|}{$2.54 \mathrm{eV}$} & \multicolumn{3}{|c|}{$2.33 \mathrm{eV}$} & \multicolumn{3}{|c|}{$1.96 \mathrm{eV}$} \\
\hline $\begin{array}{l}\text { Raman } \\
\text { shift, } \mathrm{cm}^{-1}\end{array}$ & $\begin{array}{l}\mathrm{d}_{\mathrm{t}} \\
\mathrm{nm}\end{array}$ & $\begin{array}{l}\mathrm{E} \\
\text { transition }\end{array}$ & $\begin{array}{l}\text { Raman } \\
\text { shift, } \mathrm{cm}^{-1}\end{array}$ & $\begin{array}{l}\mathrm{d}_{\mathrm{t}} \\
\mathrm{nm}\end{array}$ & $\begin{array}{l}\mathrm{E} \\
\text { transition }\end{array}$ & $\begin{array}{l}\text { Raman } \\
\text { shift, } \mathrm{cm}^{-1}\end{array}$ & $\begin{array}{l}d_{t}, \\
n m\end{array}$ & $\begin{array}{l}\mathrm{E} \\
\text { transition }\end{array}$ \\
\hline 203 & 1.15 & $\mathrm{E}_{33} \mathrm{~s}$ & 188 & 1.26 & $\mathrm{E}_{22} \mathrm{~s}$ & 196 & 1.20 & $\mathrm{E}_{11} \mathrm{M}$ \\
\hline 227 & 1.02 & $\mathrm{E}_{11}{ }^{\mathrm{M}}$ & 272 & 0.84 & $\mathrm{E}_{11}{ }^{\mathrm{M}}$ & 219 & 1.06 & $\mathrm{E}_{11}{ }^{\mathrm{M}}$ \\
\hline 260 & 0.89 & $\mathrm{E}_{11} \mathrm{M}$ & & & & 257 & 0.90 & $\mathrm{E}_{22} \mathrm{~s}$ \\
\hline 300 & 0.75 & $\mathrm{E}_{22} \mathrm{~s}$ & & & & 283 & 0.81 & $\mathrm{E}_{22} \mathrm{~s}$ \\
\hline
\end{tabular}

Comparing the RBM regions of pSWCNTs with those of LuX ${ }_{3} @$ pSWCNTs for all the excitation energies, changes in the intensity of the bands are evident. These are related to changes in the resonance conditions due to the filling that involves charge-transfer processes. The RBM bands of the Raman spectra of LuCl $1_{3} @$ pSWCNTs,LuBr $@$ pSWCNTs and LuI $@$ pSWCNTs show a small shift as compared with the spectra of the pSWCNTs (see Electronic Supporting Information Table SI1). The observed shift of breathing modes of the LuCl ${ }_{3} @$ pSWCNTs, LuBr 3 pSWCNTs and LuI 3 @pSWCNTs towards higher and lower frequencies as well as the significant changes in the intensity as compared with the pSWCNTs may be associated with a doping effect. ${ }^{30-36}$ The RBM band positions in the spectra of $\mathrm{LuI}_{3} @ \mathrm{pSWCNTs}$ are more significantly shifted than those of the $\mathrm{LuCl}_{3} @ \mathrm{pSWCNTs}$ and $\mathrm{LuBr}_{3} @$ pSWCNTs. In addition, the bands that are in resonance via $E_{11}{ }^{\mathrm{M}}$ transitions present higher shifts in the frequencies, comparing LuX ${ }_{3} @$ pSWCNTs $(\mathrm{X}=\mathrm{Cl}, \mathrm{Br}, \mathrm{I})$ with pSWCNTs, because the metallic tubes are more sensitive to doping.,

Figure $3 \mathrm{~d}-\mathrm{f}$ shows the $\mathrm{G}$ region (including the D-band and G-band) of the Raman spectra of SWCNTs excited by 2.54, 2.33 and $1.96 \mathrm{eV}$ (the positions of the G-lines are recorded in Electronic Supporting Information Table ESI2). The $\mathrm{G}^{+}$-band in Figure 3 is centred between 1588 and $1592 \mathrm{~cm}^{-1}$, 
which is typical for SWCNT bundles. The G-band frequency shifts observed between pSWCNTs and LuX 3 @pSWCNTs $(\mathrm{X}=\mathrm{Cl}, \mathrm{Br}, \mathrm{I})$ can be attributed to doping effects, as we already mentioned in the analyses of the RBM. ${ }^{30-36}$ Comparing the spectra of the pristine and filled samples, a weak shift of the G-band to both higher (blueshift) and lower (redshift) frequencies was observed. The contribution of $\mathrm{G}^{-}$metallic is the most shifted one because it is more sensitive to doping. When comparing the effects on the differentLuX $\mathrm{X}_{3} @$ pSWCNTs $(\mathrm{X}=\mathrm{Cl}, \mathrm{Br}, \mathrm{I})$ filling, as has already been observed for the RBM, the biggest changes are registered for the LuI $@$ @pSWCNTs. Moreover, in Figure 3, by comparing the same filled sample LuX $\mathrm{X}_{3} @ \mathrm{pSWCNTs}(\mathrm{X}=\mathrm{Cl}, \mathrm{Br}, \mathrm{I})$ excited with different laser energies it is possible to observe differences in the G-band line shape. It indicates that, depending on the excitation energy, different semiconducting and metallic carbon nanotubes are in resonance. In general, narrow symmetric line shapes indicate semiconducting SWCNTs while broad line shapes indicate metallic nanotubes. The line shape of the G-band is altered after filling (at the same laser excitation energy) which is a sign of charge-transfer processes. The D-band in the spectra of the pSWCNTs appears at around 1310, 1330 and $1337 \mathrm{~cm}^{-1}$ using 2.54, 2.33 and $1.96 \mathrm{eV}$ excitation energy because the frequency of the D-band is laser-energy dependent. The D-band also shifts comparing pSWCNTs with LuX $\mathrm{X}_{3} @ \mathrm{pSWCNTs}(\mathrm{X}=\mathrm{Cl}$, $\mathrm{Br}$, I) (see Electronic Supporting Information Table ESI3). Therefore, the resonance condition is changing (as we have confirmed analysing RBM and the G-band) and different SWCNT are contributing to spectra. To evaluate the number of defects in the samples, the intensity ratio between the D- and G-bands (the $A_{\mathrm{D}} / A_{\mathrm{G}}$ ratio), calculated from the integrated areas ( $A_{\mathrm{D}}$ and $\mathrm{A}_{\mathrm{G}}$, respectively), is shown in Figure 4 . Note that we propose the use of the $A_{\mathrm{D}} / A_{\mathrm{G}}$ ratio instead of the $I_{\mathrm{D}} / I_{\mathrm{G}}$ ratio because the former is less affected by the doping. ${ }^{37}$

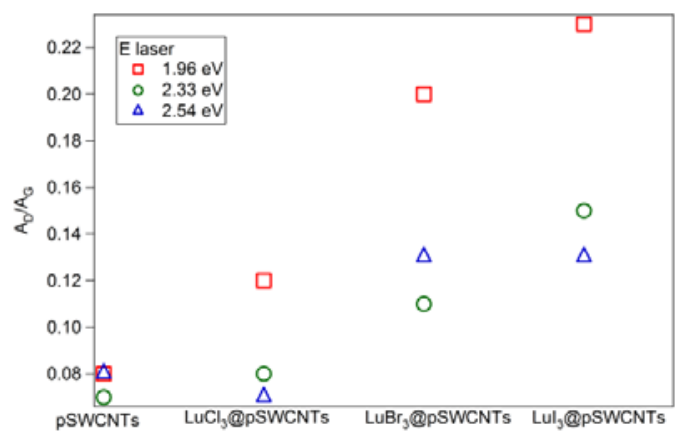

Figure 4: $A_{\mathrm{D}} / A_{\mathrm{G}}$ ratio of the pSWCNTs and LuX $3 @ \mathrm{pSWCNTs} \mathrm{samples.} \mathrm{The} \mathrm{samples} \mathrm{were} \mathrm{excited} \mathrm{using}$ $2.54 \mathrm{eV}$ (blue triangles), $2.33 \mathrm{eV}$ (green circles) and $1.96 \mathrm{eV}$ (red squares) laser energies.

Raman spectroscopy results suggest that there is a charge-transfer process involved in the filling of SWCNTs. We used in situ Raman spectroelectrochemistry to investigate in detail the changes induced in the electronic structure of SWCNTs upon endohedral functionalization. The potentialdependent Raman spectra of the pSWCNTs,LuCl $@$ SWCNT, LuBr $3 @ S W C N T$ and LuI $\mathrm{S}_{3} @$ SWCNT in the acetonitrile electrolyte solution are shown in Figure 5, 6, 7 and 8 respectively. 

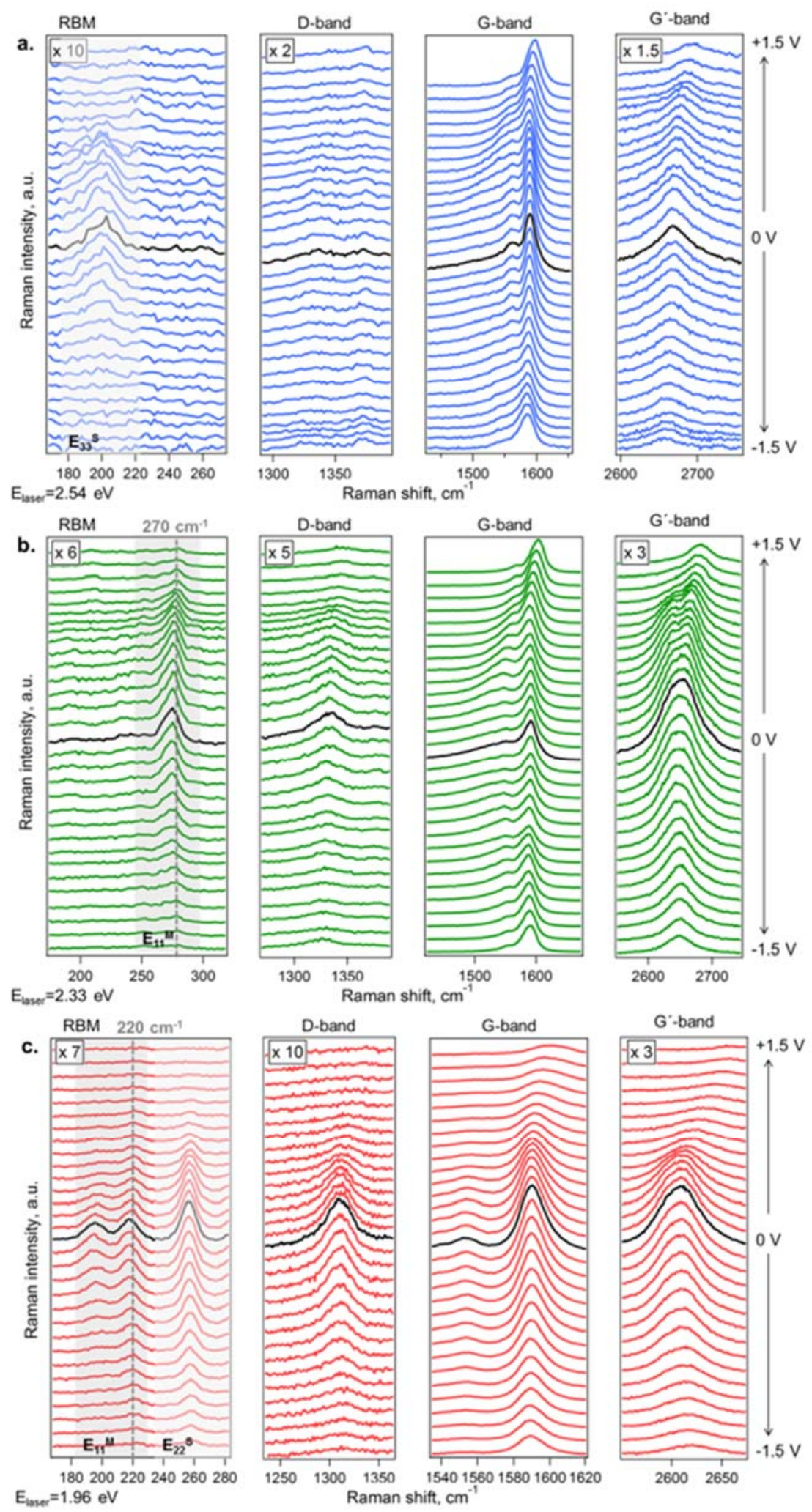

Figure 5: Raman spectra of the pSWCNTs at different potentials in the RBM, D-band, G-band, and G'band mode spectral region. The electrode potential range is from -1.5 to $1.5 \mathrm{~V}$ vs $\mathrm{Ag} / \mathrm{Ag}^{+}$(from bottom to top). The spectra in black correspond to the $0 \mathrm{~V}$ potential. The electrochemical potential change between the curves in the figure is $0.1 \mathrm{~V}$. The spectra were acquired using a. 2.54, b. 2.33 and c. 1.96 $\mathrm{eV}$ laser excitation energy. The black and grey regions in the RBM correspond to the $\mathrm{E}_{11}{ }^{\mathrm{M}}$ and $\mathrm{E}_{22} \mathrm{~S}$, $\mathrm{E}_{33} \mathrm{~S}$ electronic transitions, respectively. The spectra are offset for clarity. 

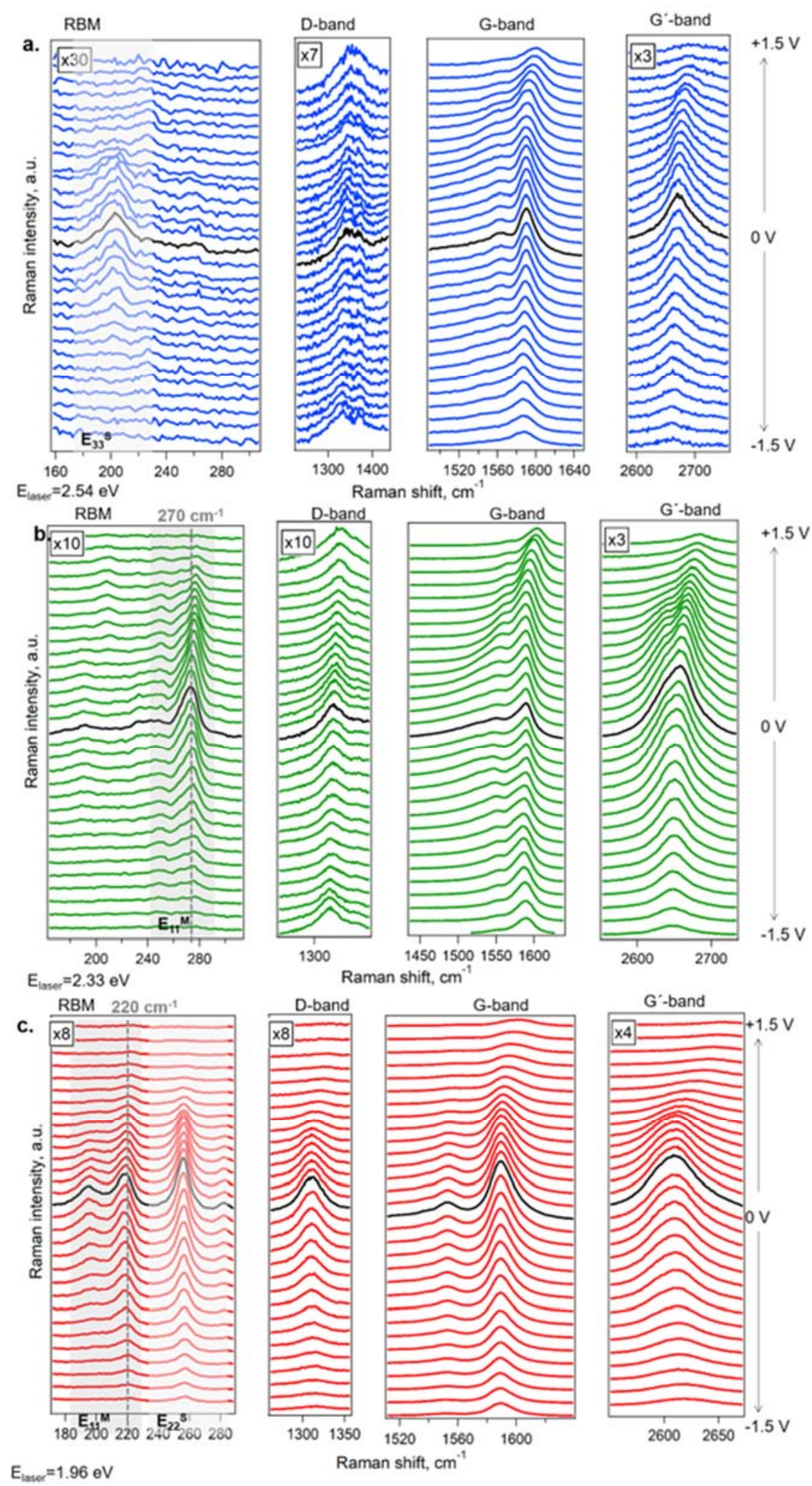

Figure 6: Raman spectra of the $\mathrm{LuCl}_{3} @$ SWCNTs at different potentials in the RBM, D-band, G-band, and $\mathrm{G}^{\prime}$-band mode spectral region. The electrode potential range is from -1.5 to $1.5 \mathrm{~V} \mathrm{vs} \mathrm{Ag} / \mathrm{Ag}^{+}$(from bottom to top). The spectra in black correspond to the $0 \mathrm{~V}$ potential. The electrochemical potential change between the curves in the figure is $0.1 \mathrm{~V}$. The spectra were acquired using a. 2.54, b. 2.33 and c. $1.96 \mathrm{eV}$ laser excitation energy. The black and grey regions in the RBM correspond to the $\mathrm{E}_{11}{ }^{\mathrm{M}}$ and $\mathrm{E}_{22} \mathrm{~s}, \mathrm{E}_{33} \mathrm{~s}$ electronic transitions, respectively. The spectra are offset for clarity. 

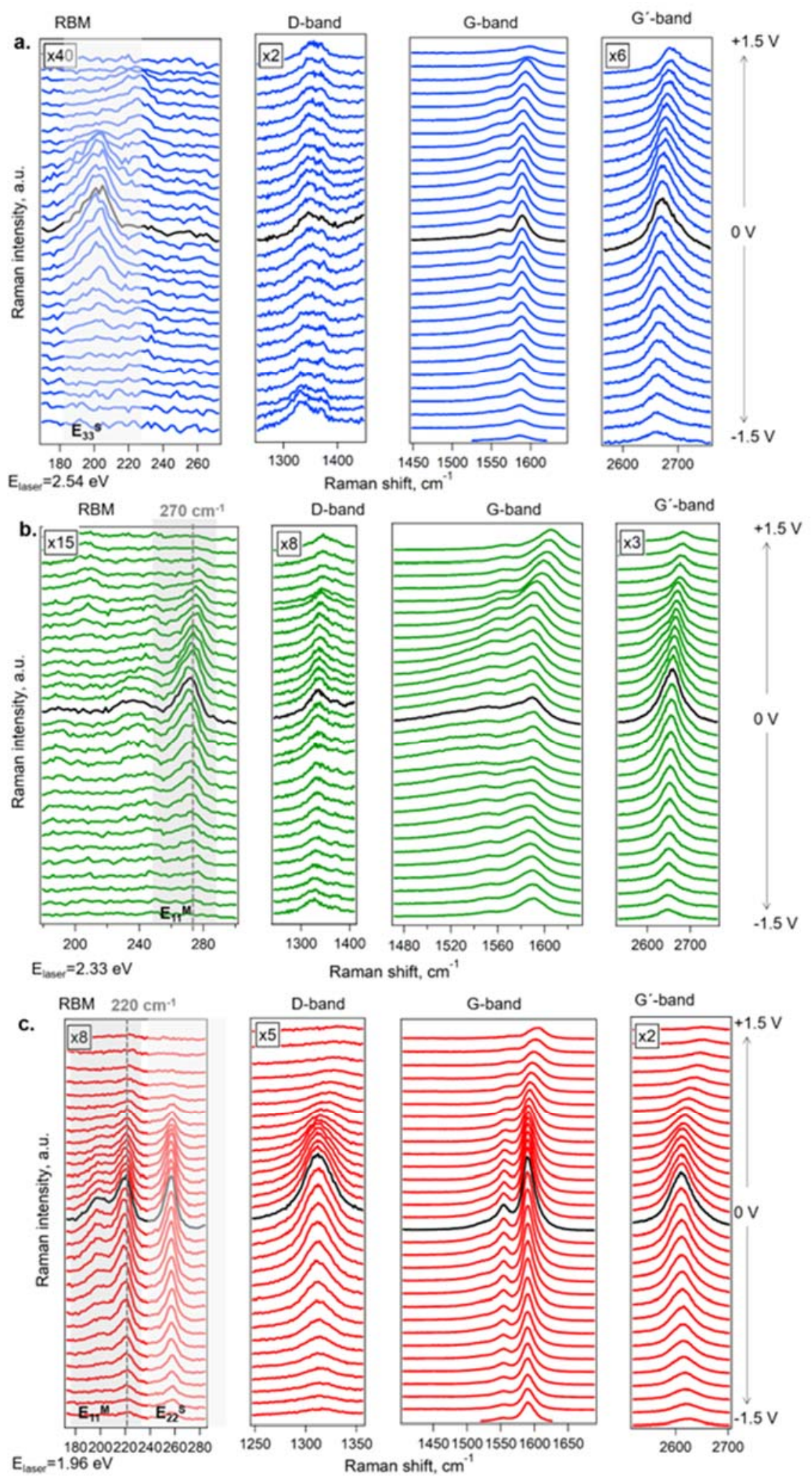

Figure 7: Raman spectra of the $\mathrm{LuBr}_{3} @$ SWCNTs at different potentials in the RBM, D-band, G-band, and $\mathrm{G}^{\prime}$-band mode spectral region. The electrode potential range is from -1.5 to $1.5 \mathrm{~V} \mathrm{vs} \mathrm{Ag} / \mathrm{Ag}^{+}$(from bottom to top). The spectra in black correspond to the $0 \mathrm{~V}$ potential. The electrochemical potential change between the curves in the figure is $0.1 \mathrm{~V}$. The spectra were acquired using a. 2.54, b. 2.33 and c. $1.96 \mathrm{eV}$ laser excitation energy. The black and grey regions in the RBM correspond to the $\mathrm{E}_{11}{ }^{\mathrm{M}}$ and $\mathrm{E}_{22} \mathrm{~s}, \mathrm{E}_{33} \mathrm{~s}$ electronic transitions, respectively. The spectra are offset for clarity. 

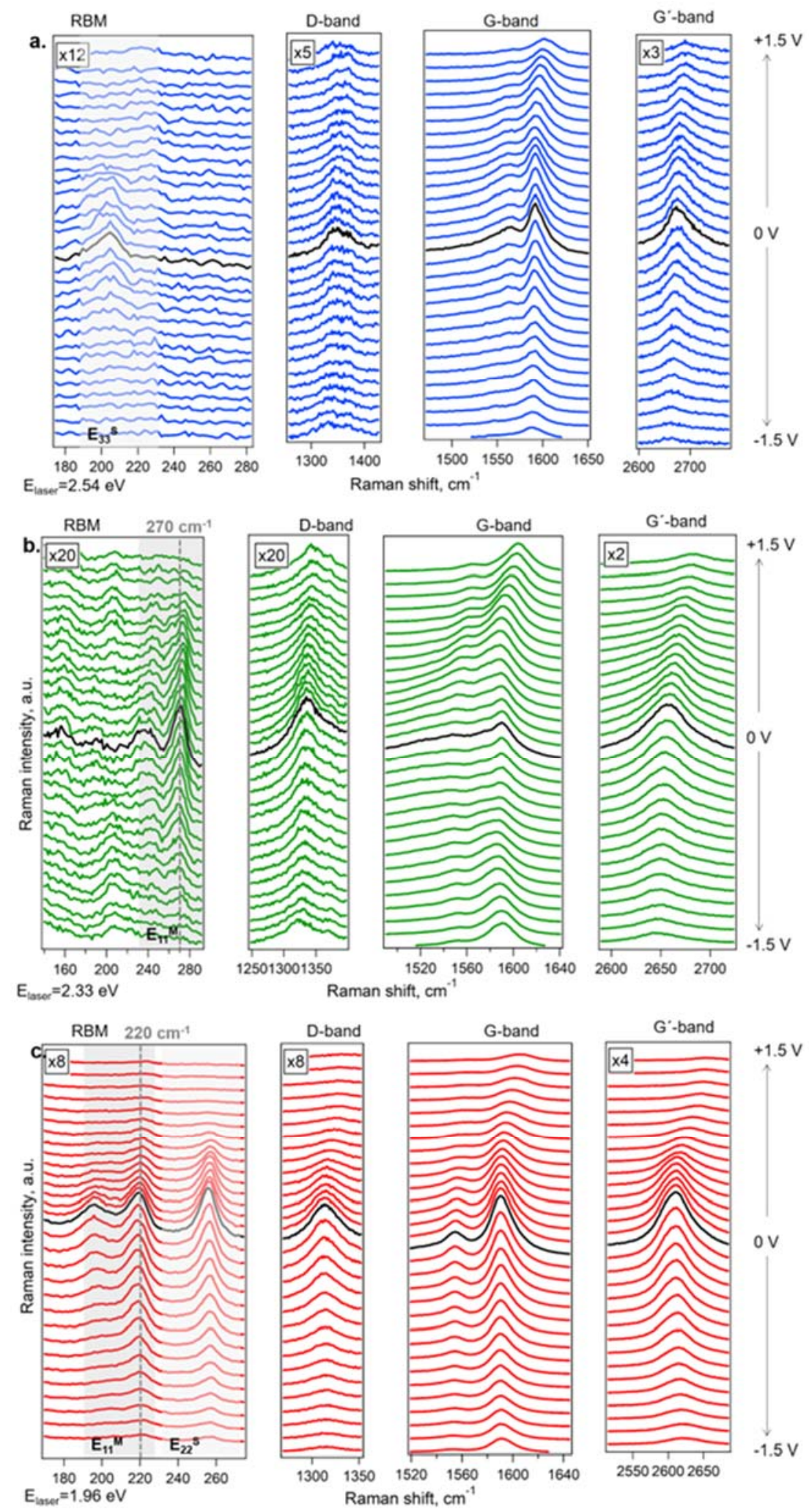

Figure 8: Raman spectra of the LuIr $@$ @SWCNTs at different potentials in the RBM, D-band, G-band, and $\mathrm{G}^{\prime}$-band mode spectral region. The electrode potential range is from -1.5 to $1.5 \mathrm{~V} \mathrm{vs} \mathrm{Ag} / \mathrm{Ag}^{+}$(from bottom to top). The spectra in black correspond to the $0 \mathrm{~V}$ potential. The electrochemical potential change between the curves in the figure is $0.1 \mathrm{~V}$. The spectra were acquired using a. 2.54, b. 2.33 and c. $1.96 \mathrm{eV}$ laser excitation energy. The black and grey regions in the RBM correspond to the $\mathrm{E}_{11}{ }^{\mathrm{M}}$ and $\mathrm{E}_{22} \mathrm{~s}, \mathrm{E}_{33} \mathrm{~s}$ electronic transitions, respectively. The spectra are offset for clarity. 
In Figures 5, 6, 7 and 8 we observe that when applying positive potentials, from $0 \mathrm{~V}$ to $+1.5 \mathrm{~V}$, in steps of $0.1 \mathrm{~V}$, the Fermi level is downshifted (p-doping, introducing holes into the $\Pi$-band). The opposite occurs when negative potentials are applied, from $0 \mathrm{~V}$ to $-1.5 \mathrm{~V}$; the electron density increases due to the upshift in the Fermi level (n-doping, introducing electrons into the $\Pi$-band). In this way, when the Fermi level reaches a van Hove singularity level, the corresponding electronic transition is blocked and the Raman signal is bleached.

Comparing Raman spectra of pSWCNT (Figure 5), LuCl $@$ SWCNT (Figure 6), $\mathrm{LuBr}_{3} @$ SWCNT (Figure 7) and LuI $@$ SWCNT (Figure 8) when potential is applied, the D-band, the G-band and the $\mathrm{G}^{\prime}$-band have similar behaviour in all the samples. In Figures 5, 6, 7, and 8, the D-mode, as explain above, is only slowly bleached as the magnitude of the electrode potential is increased, which is a consequence of blocking the resonant transition. Similar situation is happening to the $\mathrm{G}^{\prime}$ band in the Raman spectra at a frequency of $\sim 2600 \mathrm{~cm}^{-1}$ for all measured samples. The $\mathrm{G}^{\prime}$-band arises from second-order two-phonon processes, and its frequency strongly depends on the laser excitation energy. ${ }^{38}$ In addition, the $\mathrm{G}^{\prime}$-band is also sensitive to the chirality of the SWCNTs. ${ }^{39}$ In the case of nanotube bundles, the G'-band consists of contributions from many tubes. Thus, the line shape and the frequency of the $\mathrm{G}^{\prime}$-band are very difficult to analyze for samples containing SWCNT bundles. Nevertheless, the intensity of the $\mathrm{G}^{\prime}$-band is reduced with potential applied, which is in line with our previous assumption. Changes in the G-band are only small. One can trace narrowing and bleaching of the $\mathrm{G}^{-}$part of the Gband attributed to metallic tubes in the samples, which are highly sensitive to doping. ${ }^{40}$ The $\mathrm{G}^{+}$-band intensity is changed only at high doping levels because mainly semiconducting tubes contribute to the intensity of this band. At large positive potentials we observed a slight increase in the frequency of the $\mathrm{G}^{+}$band because of phonon renormalization and changes in the bond strength. ${ }^{41}$

A thorough detailed analysis of the G-band of the Raman spectra of the purified and filled SWCNTs showed that the encapsulated LuX3 salts cause doping of the host SWCNT, and the doping efficiency depends on the compound. ${ }^{32}$ Furthermore, comparing purified and filled SWCNT the changes in the intensity of the bands are evident in the RBM (Figure 3). Hence, for a more detailed analysis, the dependence of the Raman intensity of the RBM on the electrode potential was studied (see Figure 9). A difference between the profiles of the Raman intensity $\left(I_{\mathrm{RBM}}\right)$ versus potential ( $\left.E_{\text {applied }}\right)$ of metallic and semiconducting tubes was found as reported previously. ${ }^{42}$ The intensity of the band at ca. $270 \mathrm{~cm}^{-1}\left(E_{\text {laser }}=2.33 \mathrm{eV}\right)$ and the band at ca. 200 and $220 \mathrm{~cm}^{-1}\left(E_{\text {laser }}=1.96 \mathrm{eV}\right)$ is attenuated at potentials close to $0 \mathrm{~V}$ because the filling/depleting can already start when the Fermi level, $E_{\mathrm{F}}=0 \mathrm{eV}$ for metallic nanotubes. Therefore, for metallic tubes, the profile of the Raman intensity versus potential is sharp with a maximum at around $0 \mathrm{~V}$. In contrast, for semiconducting tubes, the profiles $I_{\mathrm{RBM}} \mathrm{vs}$. $E_{\text {applied }}$ of the band at ca. $200 \mathrm{~cm}^{-1}\left(E_{\text {laser }}=2.54 \mathrm{eV}\right)$ and the band at $260 \mathrm{~cm}^{-1}\left(E_{\text {laser }}=1.96 \mathrm{eV}\right)$ exhibit a plateau close to $0 \mathrm{~V}$. The Raman intensity is not significantly attenuated close to $0 \mathrm{~V}$, it starts changing 
only after the electrode potential reaches the first van Hove singularity (vHs). Thus, we can distinguish between metallic and semiconducting nanotubes. ${ }^{42}$ These assignments obtained with the $I_{\text {RBM }}$ vs. $E_{\text {applied }}$ profiles are in agreement with the results obtained by assigning the electronic transitions in Figure 3 using the Kataura plot.
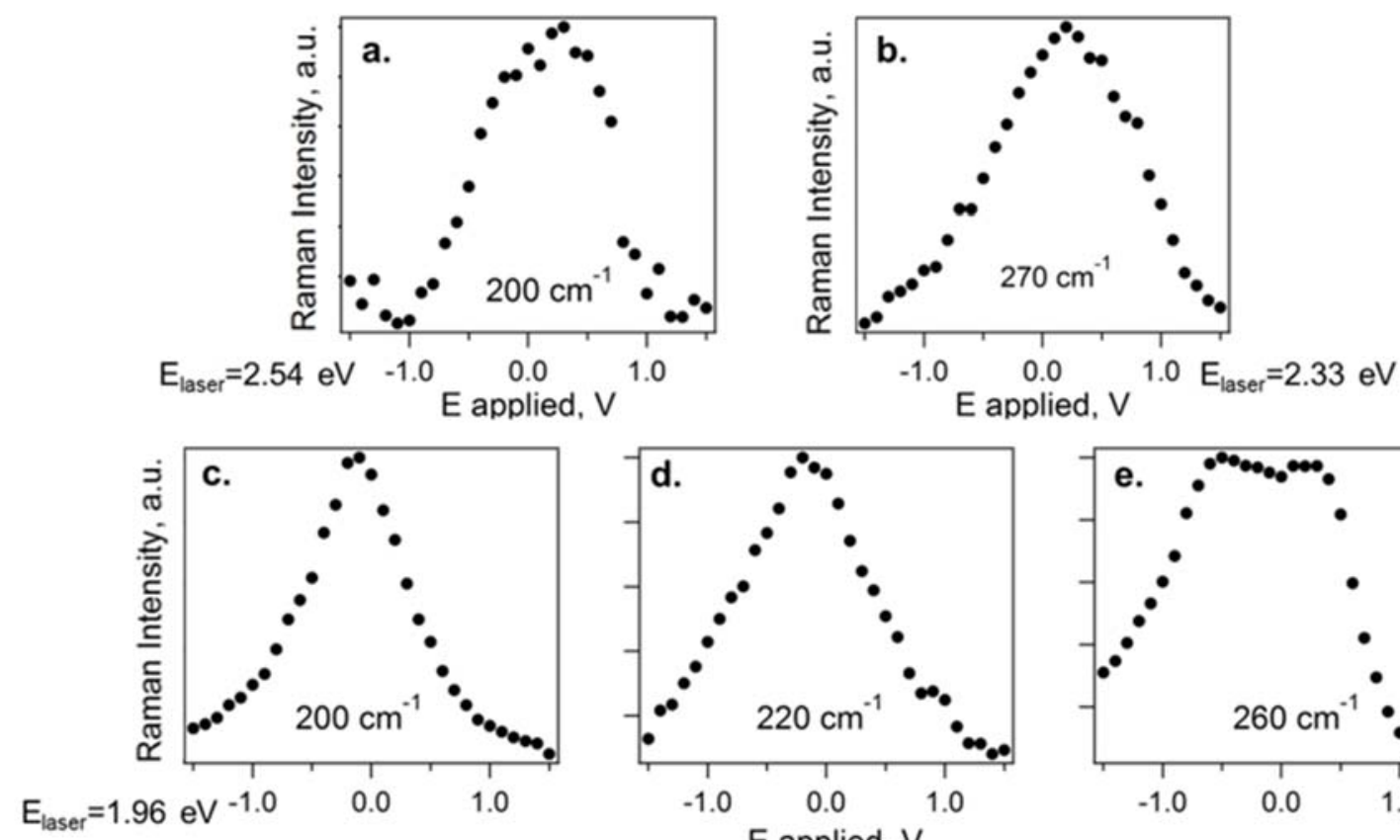

Figure 9: $I_{\text {RBM }}$ Raman intensity of the RBM vs. potential profile of pSWCNTs in resonance at $2.54 \mathrm{eV}$. a. band $200 \mathrm{~cm}^{-1}$ (semiconducting tubes $E_{33} \mathrm{~S}$ ), at $2.33 \mathrm{eV}$. b. band $270 \mathrm{~cm}^{-1}$ (metallic tubes $E_{11}{ }^{\mathrm{M}}$ ) and in resonance at $1.96 \mathrm{eV}$. c. bands 200 and $220 \mathrm{~cm}^{-1}$ (metallic tubes $E_{11}{ }^{\mathrm{M}}$ ) and d. band $260 \mathrm{~cm}^{-1}$ (semiconducting tubes $E_{33} \mathrm{~S}$ ).

As shown above, metallic SWCNTs are more sensitive to doping. For this reason, the $I_{\text {RBM }}$ vs. $E_{\text {applied }}$ profiles of the bands in resonance via the $E_{11}{ }^{\mathrm{M}}$ transition (grey vertical dashed line in Figure 5, 6,7 , and 8), were investigated in detail for purified SWCNTs and filled samples.

Here we report the study of the changes in RBM intensity with applied potential for the first time for endohedral functionalized SWCNT. The profiles of the RBM intensity for pSWCNTs and $\mathrm{LuX}_{3} @$ pSWCNTs $(\mathrm{X}=\mathrm{Cl}, \mathrm{Br}, \mathrm{I})$ samples, shown in Figures 10 and 11, were obtained using 2.33 and $1.96 \mathrm{eV}$ laser excitation energy, respectively. In these figures, we observe that the Raman intensity reaches a maximum at a given potential indicating the optimum conditions for resonance scattering. We found that the maximum of the Raman RBM band intensity for the metallic transition versus applied potential is shifted for the filled samples in comparison with pSWCNTs. 

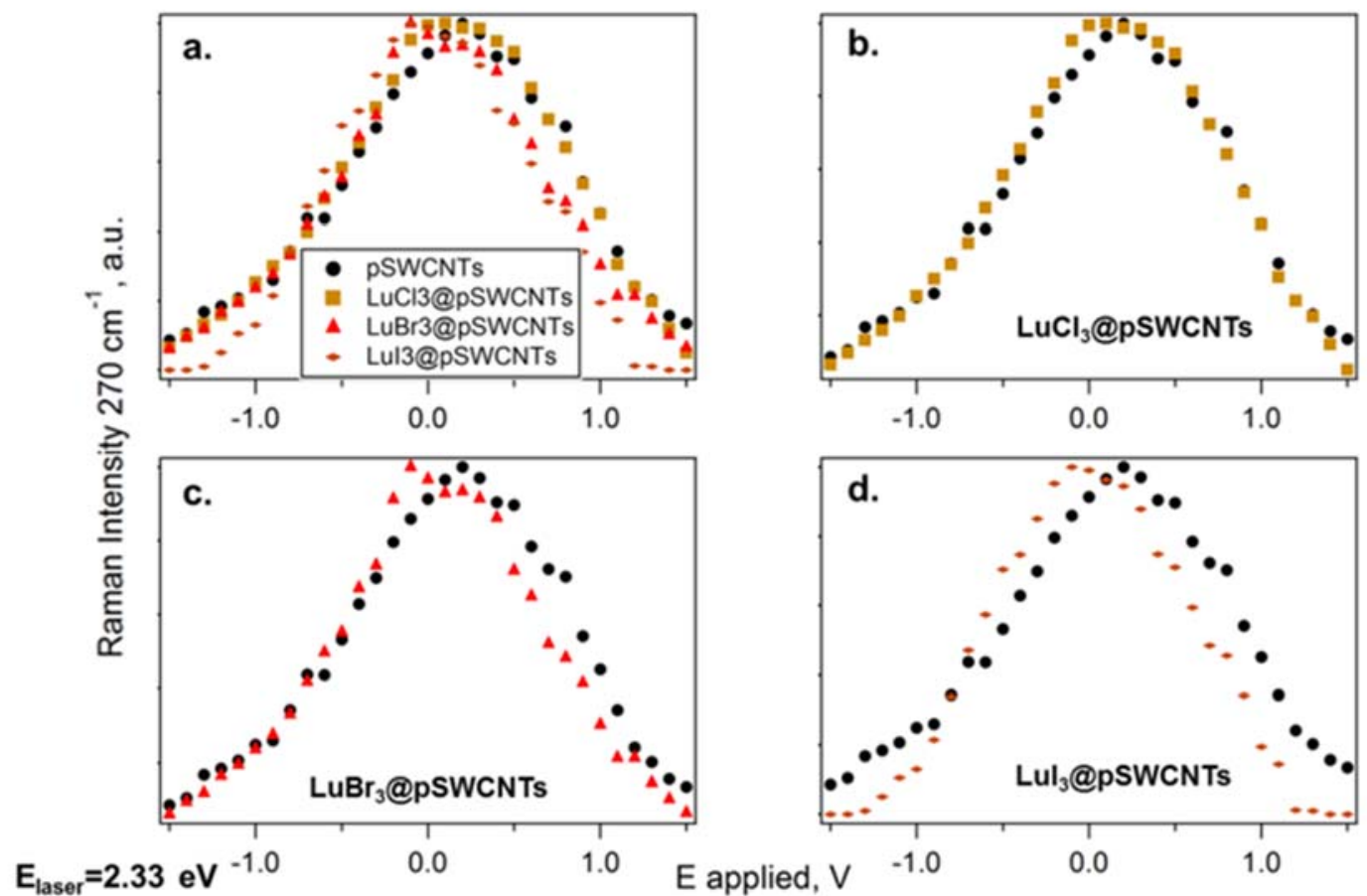

Figure 10: $I_{\text {RBM }}$ Raman intensity of the band at $270 \mathrm{~cm}^{-1}$ of metallic tubes in resonance via $E_{11}{ }^{\mathrm{M}}$ vs. potential profile of pSWCNTs (black circles), LuCl $@$ pSWCNTs (yellow squares),_LuBr $3 @ p S W C N T s$ (red triangles) and LuI 3 pSWCNTs (brown dots) in resonance at $2.33 \mathrm{eV}$.
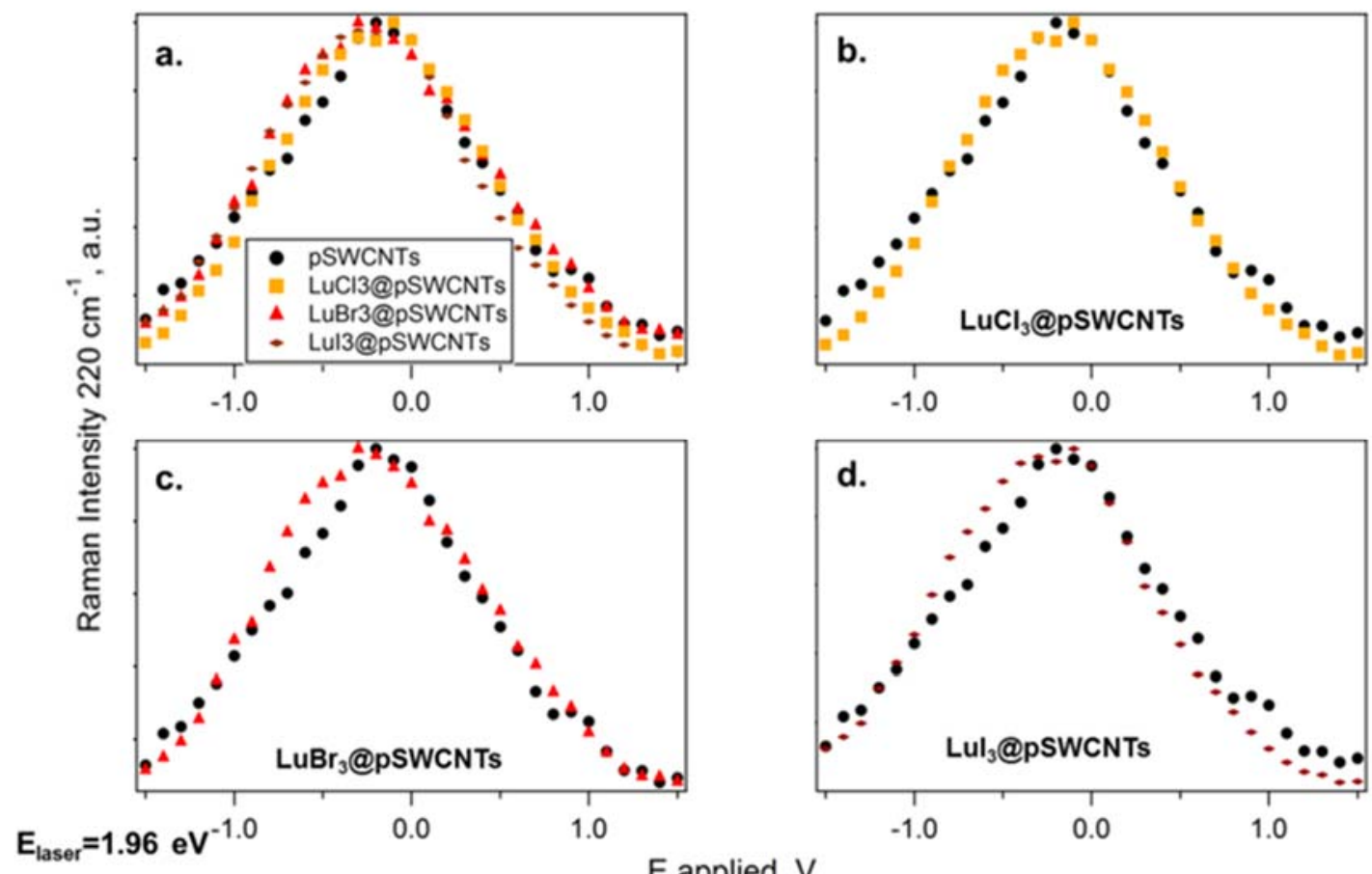

Figure 11: $I_{\text {RBM }}$ Raman intensity of the band at $220 \mathrm{~cm}^{-1}$ metallic tubes in resonance via $\mathrm{E}_{11}{ }^{\mathrm{M}}$ vs. potential profile of pSWCNTs (black circles), LuCl $@$ pSWCNTs (yellow squares), LuCl ${ }_{3} @$ pSWCNTs (red triangles) and LuI @pSWCNTs (brown dots) in resonance at $1.96 \mathrm{eV}$. 
In the present work, we focus on monitoring the changes in the RBM. We show that studying the development of the RBM intensities in resonance via the electronic transition $\mathrm{E}_{11}{ }^{\mathrm{M}}$ during electrochemical charging we have been able to determine the type of doping (n-/p-). For both laser energies used, the profile of the $I_{\mathrm{RBM}}$ in Figures 10 and 11 is shifted for $\mathrm{LuCl}_{3} @$ pSWCNTs (yellow squares),LuBr 3 pSWCNTs (red triangles) and greatly in the case of LuI ${ }_{3} @$ pSWCNTs (brown dots), in relation to pSWCNTs (black circles) and especially using $2.33 \mathrm{eV}$ excitation energy because there are more metallic SWCNTs in resonance with this $E_{\text {laser. }}$ This indicates that doping is induced on the SWCNTs by the filling. It is more remarkable for LuI ${ }_{3} @$ pSWCNTs. As seen in Figures 9 and 10, for $\mathrm{LuI}_{3} @$ pSWCNTs (brown dots) it is necessary to apply a large negative potential to reach the maximum peak of Raman intensity, suggesting that the SWCNTs are $p$-doped and a downshift of the Fermi level occurs, while the electronic density of states (DOS) remains untouched. In this way, the valence band vHs is emptied (depleted) and it is necessary to apply a more negative potential to reach the neutral point. With this new in situ spectroelectrochemical method we successfully study the behaviour of filled SWCNT during doping and we are able to distinguish between $n$ - or $p$ - doping. This method can constitute a routine test to estimate the electronic effects of functionalization being also used to predict the electronic properties of functionalized SWCNT in devices as it was confirmed in previous work for covalent functionalized SWCNT. ${ }^{4}$ It is very beneficial since most of the functionalization procedures for nanocarbon structures are sensitive to the electronic properties of the SWCNTs and can also lead to significant changes in their electronic structure. These results validate the in situ Raman spectroelectrochemistry as a unique tool box for predicting the electronic properties of endohedral functionalized SWCNTs for future optoelectronic applications.

\section{Conclusions}

To summarize, in the present work, the role of steam-treatment time on raw arc-discharge SWCNTs has been investigated for the first time. We have shown that steam can remove the amorphous carbon and graphitic shells coating the catalytic particles. Subsequent treatment with $\mathrm{HCl}$ removes the catalytic nanoparticles themselves. All the samples of purified SWCNTs present a lower metal content than in the raw material. The lowest number of catalytic particles is achieved after a $4 \mathrm{~h}$ steam treatment followed by $\mathrm{HCl}\left(2.9 \mathrm{wt} \%\right.$ of Fe). The steam-treated samples present a continuous decrease in the $I_{\mathrm{D}} / I_{\mathrm{G}}$ ratio upon increasing the steam-treatment time, being an indication of the removal of the amorphous carbon and the most defective SWCNTs. In contrast to other purification treatments, steam does not modify the nanotube tubular structure and avoids the formation of defects and functional groups. After $4 \mathrm{~h}$ steam treatment, the SWCNTs are shorter (457 nm median length) than the raw SWCNTs $(537 \mathrm{~nm}$ median length). Besides, the $\mathrm{LuX}_{3} @ S W C N T(\mathrm{X}=\mathrm{Cl}, \mathrm{Br}, \mathrm{I})$ nanostructures were synthesized using molten-phase capillary filling of the internal channels of arc-discharge single-walled nanotubes with the melts of lutetium(III) chloride, lutetium(III) bromide and lutetium(III) iodide. Direct evidence of 
the confinement of the investigated materials inside carbon nanotubes was provided by SEM imaging in transmission mode. The yield of the encapsulation was 7.1, 9.8 and 7.8 wt $\%$ for $\mathrm{LuCl}_{3} @ \mathrm{SWCNTs}$, LuBr$_{3} @$ SWCNTs and LuI ${ }_{3} @$ SWCNTs, respectively. The Raman spectra suggest that the lutetium(III) halides encapsulated inside the SWCNTs channels lead to a shift of the Fermi level. We developed a new method using in situ Raman spectroelectrochemistry to study the doping effect related to the encapsulation of these compounds in detail. The results show that the lutetium(III) halides display acceptor behaviour with the charge transfer from the carbon nanotube walls to the incorporated onedimensional nanocrystals. This study opens up new possibilities of applications of LuX $\mathrm{X}_{3} @$ SWCNTs in electronics.

\section{Acknowledgements}

The research leading to these results has received funding from the People Programme (Marie Curie Actions) of the European Union's Seventh Framework Programme FP7 under REA grant agreement 290023 (RADDEL). We also acknowledge financial support from the Czech science foundation (2008633X) and the Spanish Ministry of Economy and Competitiveness through the 'Severo Ochoa' Programme for Centres of Excellence in R\&D (SEV-2015-0496, ICMAB and SEV-2017-0706, ICN2).

\section{References}

1. M. Kierkowicz, E. Pach, A. Santidrián, E. Tobías-Rossell, M. Kalbac, B. Ballesteros, G. Tobias, ChemNanoMat, 2016, 2, 108.

2. L. D' Accolti, A. Gajewska, M. Kierkowicz, M. Martincic, A. Nacci, S. Sandoval B. Ballesteros, G. Tobias, T. Da Ros, C. Fusco, Nanomaterials, 2018, 8, 137.

3. C. Spinato, A. Perez Ruiz de Garibay, M. Kierkovwicz, E. Pach, M. Martincic, R. Klippstein, M. Bourgognon, J. Tzu-Wen Wang, C. Menard-Moyon, K. T. Al-Jamal, B. Ballesteros, G. Tobias, A. Bianco, Nanoscale, 2016, 8, 12626.

4. J. M. Gonzalez-Dominguez, A. Santidrian, A. Criado, C. Hadad, M. Kalbac, T. Da Ros, Chemistry - A European Journal, 2015, 21, 18631.

5. A. Santidrian, J. M. Gonzalez-Dominguez, V. Diez-Cabanes, J. Hernandez-Ferrer, W. K. Maser, Ana M. Benito, A. Ansón-Casaos, J. Cornil, T. Da Ros, M. Kalbac, Physical Chemistry Chemical Physics, 2019, 21, 4063.

6. M. Sauer, A. Briones-Leon, T. Saito, K. Yanagi, K. Sachulte, T. Pichler, H. Shiozawa, Physica status solidi B, 2015, 11, 2546.

7. M.V. Kharlamova, Applied Physics A-Materials Science \& Processing, 2016, 122, 791.

8. M. Endo, M. S. Strano, P. M. Ajayan, Ed. A. Jorio, G. Dresselhaus and M. Dresselhaus, Springer-Verlag GmbH, Heidelberg, 2008, 111, 13.

9. D. Iglesias, M. Melchiona, Catalysts, 2019, 9, 128. 
10. A. Setaro, Journal of Physics: Condensed Matter, 2017, 29, 423003.

11. M.V. Kharlamova, Progress in Materials Science, 2016, 77, 125-211.

12. T. Takenobu, T. Takano, M. Shiraishi, Y. Murakami, M. Ata, H. Kataura, Y. Achiba, Y. Iwasa, Nature Materials, 2003, 2, 683.

13. S.B. Fagan, A.G.S. Filho, J.M. Filho, P. Corio, M.S. Dresselhaus, Chemical Physics Letters, 2005, 406, 54.

14. J. Lee, J. Lee, J. Yang, W. Yi, Journal of Nanoscience and Nanotechnology, 2019, 19, 6144.

15. C. Thomsen, S. Reich, Topics in Applied Physics, 2007, 108, 115.

16. A. Jorio, M. S. Dresselhaus, R. Saito, G. Dresselhaus, John Wiley \& Sons, 2011.

17. M. A. Pimenta, A. Marucci, S. A. Empedocles, M. G. Bawendi, E. B. Hanlon, A. M. Rao, P. C. Eklund, R. E. Smalley, G. Dresselhaus, M. S. Dresselhaus, Physical review B, 1998, 58, R16016.

18. M.S. Dresselhaus, G. Dresselhaus, A. Jorio, A.G. Souza Filho, R. Saito, Carbon, 2002, 40, 2043.

19. J. Maultzsch, S. Reich, C. Thomsen, Physical review B, 2001, 64, 121407.

20. L. Kavan, L. Dunsch, Topics in Applied Physics, 2008, 111, 567.

21. L. Kavan, L. Dunsch, ChemPhysChem, 2011, 12, 47.

22. B. Nikolić, I. Milošević and M. Damnjanović, The Journal of Physical Chemistry C, 2014, 118, 20576-20584.

23. T. Julie, W. Wang, R. Klippstein, M. Martincic, E. Pach, R. Feldman, M. Sefl, Y. Michel, D. Asker, J. K. Sosabowski, M. Kalbac, T. Da Ros, C. Ménard-Moyon, A. Bianco, I. Kyriakou, D. Emfietzoglou, J. C. Saccayini, B. Ballesteros, K. T. Al-Jamal, G. Tobias, ACS Nano, In press.

24. B. Ballesteros, G. Tobias, L. Shao, E. Pellicer, J. Noqués, E. Mendoza, M.L. Green, Small, 2008, 9, 1501.

25. M. Kierkowicz, J.M. Gonzalez-Dominguez, E. Pach, S. Sandoval, B. Ballesteros, T. Da Ros, G. Tobias, ACS Sustainable Chemistry \& Engineering, 2017, 5, 2501.

26. M. Kierkowicz, E. Pach, A. Santidrian, S. Sandoval, G. Gonçalves, E. Tobías-Rossell, M. Kalbac, B. Ballesteros, G. Tobias, Carbon, 2018, 139, 922.

27. L. Shao, G. Tobias, Y. Huh, M. L. H. Green, Carbon, 2006, 44, 2855.

28. H. Telg, J. Maultzsch, S. Reich, F. Hennrich, C. Thomsen, Physical Review Letters, 2004, 93, 177401.

29. P.T.Araujo, P.B.C.Pesce, M.S.Dresselhaus, K.Sato, R.Saito, A.Jorio, Physica E, 2010, 42, 1251.

30. A. A. Eliseev, L.V. Yashina, N.I. Verbitskiy, M. M. Brzhezinskaya, M. V. Kharlamova, M. V. Chernysheva, A. V. Lukashin, N. A. Kiselev, A.S. Kumskov, B.Freitag, A.V. Generalov, A. S. Vinogradou, Y. V. Zubavichus, E.Kleimenov, M. Nachtegall, Carbon, 2012, 50, 4021. 
31. M. V. Kharlamova, A. A. Volykhov, L. V. Yashina, A. V. Egorov, A. V. Lukashin, Journal of Materials Science, 2015, 50, 5419.

32. M. V. Kharlamova, Applied Physics A, 2015, 118, 27.

33. Y. Almadori, G. Delport, R. Chambard, L. Orcin-Chaix, A. C. Selvati, N. Izard, A. Belhboub, R. Aznar, B. Jousselme, S. Campidelli, P. Hermet, R. Le Parc, T. Saito, Y. Sato, K. Suenaga, P. Puech, J. S. Lauret, G. Cassabois, J.-L. Bantignies, L. Alvarez, Carbon, 2019, 149, 772-780.

34. S. Sandoval, G. Tobias, E. Flahaut, Inorganic Chimica Acta, 2019, 492, 66.

35. A.A. Tonkikh, V.I. Tsebro, E.A. Obraztsova, D.V. Rybkovskiy, A. S. Orekhov, I.I. Kondrashov, E.I. Kauppinen, A. L. Chuvilin, E. D. Obraztsova, Nanoscale, 2019, 11, 6755.

36. M. V. Kharlamova, L. V. Yashina, A. V. Lukashin, Journal of Materials Science, 2013, 48, 8412.

37. M. Kalbac, L. Kavan, Carbon, 2010, 48, 832.

38. M. Pimenta, E. B. Hanlon, A. Marucci, P. Corio, S. D. M. Brown, S. Empedocles, M. G. Bawendi, G. Dresselhaus, M. S. Dresselhaus, Brazilian Journal of Physics, 2000, 30, 423.

39. M. S. Dresselhaus, G. Dresselhaus, A. Jorio, Journal of Physical Chemistry C, 2007, 111, 17887.

40. M. Kalbac, L. Kavan, L. Dunsch, M.S. Dresselhaus, NanoLetters, 2008, 8, 1257.

41. M. Kalbac, H. Farhat, L. Kavan, J. Kong, M. S. Dresselhaus, M. S. NanoLetters, 2008, 8, 3532 .

42. M. Kalbac, H. Farhat, L. Kavan, J. Kong, K. Sasaki, R. Saito, M. S. Dresselhaus, ACSNano, 2009, 3, 2320. 